\title{
Cervical laser vaporization for women with cervical intraepithelial neoplasia-3
}

OYukiharu Todo, Hiroyuki Yamazaki, Chisa Shimada, Hidenori Kato.

Division of Gynecologic Oncology, Hokkaido Cancer Center, 4-2 Kikusui, Shiroishi-ku, Sapporo 003-0804, Japan

Objective: This study evaluated outcomes of laser vaporization of the cervix for women with cervical intraepithelial neoplasia (CIN)-3.

Methods: We retrospectively reviewed 161 consecutive patients with CIN3 who were treated with cervical laser vaporization between January 2008 and December 2012. At each follow-up visit, histologically confirmed CIN2, CIN3, and invasive carcinoma were defined as treatment failures, as were high-grade squamous intraepithelial lesion (HSIL) or atypical squamous cells that cannot exclude HSIL (ASCH) with subsequent treatment or lost to follow-up. Treatment failure rates were estimated by the Kaplan-Meier method.

Results: Patients' median age was 31 years old. Median follow-up period was 67 months (interquartile range: 52-74 months). Over 5 years, $70.8 \%$ continued their follow-up visits, but significantly more patients aged $\geq 35$ years did so (86.4\%) than did those aged $\leq 34$ years $(61.8 \%, P=0.0009)$. Treatment failure was observed in $14(8.7 \%)$ patients, 1 of whom progressed to invasive cancer $(0.6 \%)$. Cumulative treatment failure rates were 1-year: 5.1\%, 2-year: $6.4 \%$, and 5-year: $9.5 \%$. Among patients who suffered treatment failures, $57.1 \%$ initial failures occurred within the first year and $71.4 \%$ within the first 2 years.

Conclusions: The importance of continuing follow-up visits after 5 years should be emphasized to patients with CIN3 who are candidates for cervical laser vaporization, especially those aged $\leq 34$ years.

\begin{tabular}{lc}
\hline Characteristic & Value \\
\hline Age (y), median (range) & $31(19-67)$ \\
Age (y), $n$ (\%) & $11(6.8 \%)$ \\
$<24$ & $47(29.2 \%)$ \\
$25 \sim 29$ & $44(27.3 \%)$ \\
$30 \sim 34$ & $39(24.2 \%)$ \\
$35 \sim 39$ & $16(9.9 \%)$ \\
$40 \sim 44$ & $4(2.5 \%)$ \\
$45<$ & $1(0-7)$ \\
Gravida, median (range) & $0(0-5)$ \\
Para, median (range) & \\
Marital status & $66(41.0 \%)$ \\
married & $10(6.2 \%)$ \\
single, divorced & $85(52.8 \%)$ \\
single, never married & \\
Smoking habit & $41(25.5 \%)$ \\
yes & $115(71.4 \%)$ \\
no & $5(3.1 \%)$ \\
unkown & \\
High-risk HPV status, $n$ (\%) & $110(68.3 \%)$ \\
positive & $12(7.5 \%)$ \\
negative & $39(24.2 \%)$ \\
unknown & Table 1. Patient charcteristics of 161 patients in the study population
\end{tabular}

The cumulative 5-year DFS rate was $90.5 \%$ (Figure 1). Treatment failure was observed in 14 (9\%) patients (Table 3), 1 of whom $(0.6 \%)$ progressed to invasive cancer Cumulative 1-year, 2-year, and 5-year treatment failure rates were $5.1 \%$, $6.4 \%$, and $9.5 \%$, respectively. There was no significant difference in initial treatment failure rates between patients aged $\leq 34$ years and those aged $\geq 35$ years $(6.9 \%$ vs. $11.9 \%, P=0.28)$.

\section{Disease-free survival rate (\%)}

$$
\begin{array}{r}
100 \\
98 \\
96 \\
94 \\
92 \\
90 \\
88 \\
86 \\
84 \\
82 \\
80
\end{array}
$$

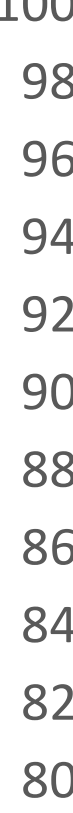

Patients' characteristics are shown in Table 1. Traceability data are shown in Table 2. The median follow-up period was 67 months after cervical laser vaporization (interquartile range: 5274 months). Lost to follow-up rates were $7.4 \%$ within the first year, $14.9 \%$ within 2 years, and $16.1 \%$ within 3 years. Of the 114 patients (70.8\%) who kept up regular hospital visits over 5 years or longer, a significantly higher percentage of those aged $\geq 35$ years $(86.4 \%)$ did so than the $\leq 34$-year group (61.8\%; $P=0.0009$ ). Those who did not maintain long-term ( $\geq 5$ years) hospital visits were, by age group, $\geq 24$ years: $54.5 \%(6 / 11) ; 25-29$ years: $38.3 \%(18 / 47)$; $30-34$ years: 34.1\% (15/44); 35-39 years: 15.4\% (6/39); 40-44 years: 12.5\% (2/16) \%; and $\geq 45$ years: $0 \%(0 / 4)$.

\begin{tabular}{cccccccc}
\hline & $<24$ yo & $25-29$ yo & $30-34$ yo & $35-39$ yo & $40-44$ yo & $>45$ yo & total \\
\hline 0-11 (month) & 1 & 4 & 3 & 3 & 1 & 0 & 12 \\
$12-23$ (month) & 2 & 4 & 6 & 0 & 0 & 0 & 12 \\
$24-35$ (month) & 0 & 1 & 0 & 0 & 1 & 0 & 2 \\
$36-47$ (month) & 1 & 4 & 3 & 0 & 0 & 0 & 8 \\
$48-59$ (month) & 1 & 5 & 3 & 3 & 0 & 0 & 12 \\
$60-71$ (month) & 4 & 14 & 15 & 14 & 5 & 1 & 54 \\
$72-$ (month) & 2 & 15 & 14 & 19 & 9 & 3 & 61 \\
\hline total & 11 & 47 & 44 & 39 & 16 & 4 & 161 \\
\hline
\end{tabular}

Table 2. Relationship between age at the initial laser vaporization and last follow-up visit

Among patients with treatment failure, initial failure occurred in $57.1 \%(8 / 14)$ within the first 1 year and 71.4\% (10/14) within the first 2 years (Table 3). Among patients with treatment failure who were treated at the age of $\leq 34$ years, initial failure occurred in $71.5 \%(5 / 7)$ within the first 1 year and 85.7\% (6/7) within the first 2 years.

\begin{tabular}{cccccccc}
\hline & $<24$ yo & $25-29$ yo & $30-34$ yo & $35-39$ yo & $40-44$ yo & $>45$ yo & total \\
\hline $0-11$ (month) & 1 & 2 & 2 & 2 & 1 & 0 & 8 \\
$12-23$ (month) & 0 & 1 & 0 & 1 & 0 & 0 & 2 \\
$24-35$ (month) & 0 & 0 & 1 & 0 & 0 & 0 & 1 \\
$36-47$ (month) & 0 & 0 & 0 & 0 & 2 & 0 & 2 \\
$48-59$ (month) & 0 & 0 & 0 & $1^{+}$ & 0 & 0 & 1 \\
$60-71$ (month) & 0 & 0 & 0 & 0 & 0 & 0 & 0 \\
$72-$ (month) & 0 & 0 & 0 & 0 & 0 & 0 & 0 \\
\hline total & 1 & 3 & 3 & 4 & 3 & 0 & 14 \\
\hline
\end{tabular}

yo, years old; + , invasive squamous cell carcinoma

Table 3. Initial treatment failure period by age 